\title{
Locality-Sensitive Support Vector Machine by Exploring Local Correlation and Global Regularization
}

\author{
Guo-Jun Qi ${ }^{\dagger}$, Qi Tian ${ }^{\ddagger}$ and Thomas Huang ${ }^{\dagger}$ \\ Beckman Institute, University of Illinois at Urbana-Champaign ${ }^{\dagger}$ \\ Department of Computer Science, University of Texas at San Antonio \\ $\left\{\right.$ qi4,t-huang1\}@illinois.edu ${ }^{\dagger}$, qitian@cs.utsa.edu ${ }^{\ddagger}$
}

\begin{abstract}
Local classifiers have obtained great success in classification task due to its powerful discriminating ability on local regions. However, most of them still have restricted generalization in twofold: (1) each local classifier is sensitive to noise in local regions which leads to overfitting phenomenon in local classifiers; (2) the local classifiers also ignore the local correlation determined by the sample distribution in each local region. To overcome the above two problems, we present a novel locality-sensitive support vector machine (LSSVM) in this paper for image retrieval problem. This classifier applies locality-sensitive hashing (LSH) to divide the whole feature space into a number of local regions, on each of them a local model can be better constructed due to smaller within-class variation on it. To avoid these local models from overfitting into localitysensitive structures, it imposes a global regularizer across local regions so that local classifiers are smoothly glued together to form a regularized overall classifier. local correlation is modeled to capture the sample distribution that determines the locality structure of each local region, which can increase the discriminating ability of the algorithm. To evaluate the performance, we apply the proposed algorithm into image retrieval task and competitive results are obtained on the real-world web image data set.
\end{abstract}

\section{Introduction}

Generic image annotation has attracted many attentions in computer vision community due to booming web images in many photo-sharing websites such as Flickr and Picasa [14][6][12]. To efficiently share these images among friends and other users in the social community, it is intensively desired to efficiently organize these images on the semantic level according to their content. As an indispensable ingredient, image categorization is an important step to analyze the image semantics which assigns a set of concepts

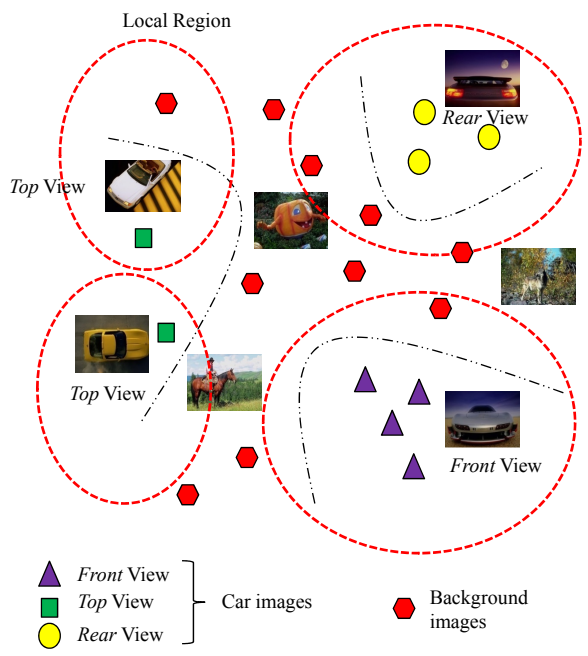

Figure 1. An example of locality-sensitive classifier for categorizing car images. Car images contain either rear view, top view or front view for example. The whole space is divided into a set of local regions marked in red circles, on each of which a local classifier is learned. Since within-class variance is alleviated in each local region so that better performance can be obtained by local learners with a simplified classification boundary.

to these images.

Many advanced algorithms have been proposed to approach practical image classification. However, due to its intrinsic complexity, it is still challenging especially for classifying generic images. As compared with many other classification problems such as shape and face recognition, generic image classification involves a wide range of image sources taken by millions of users, including both highquality photos taken by professional photographer and lowquality amateur photos captured by mobile phone, as well as the photos taken world-widely with quite different cultural and geographical background. It imposes great challenges on learning classifiers to bridge such diversified gap between low-level image signals processible by comput- 
ers and high-level semantic concepts perceptible by humankind. In other words, feature vectors extracted from an identical image category are usually scattered into a feature spaces diversely and the classification boundaries between different categories are usually highly complex due to large intra-class variances and vague inter-class discrimination. See Figure 1 as an example of categorizing car images. As shown, car images have either rear, top, or front views with highly variants. Such large variances often lead to much complex classification boundaries in practice, and more capable classifiers are required for satisfactory categorization performance. A single classifier globally constructed over the whole space may be inadequate to capture all the variants of feature representations. On the contrary, it is promising to divide the whole space into a set of locality-sensitive regions on each of which a local classifier is learned for image categorization. Since each obtained locality region only contains images with relatively smaller variances, the original complex boundaries can be factorized into much simpler ones which are tractable by local classifiers. For example, in Figure 1, the feature spaces are divided into a set of local regions with relatively consistent car views that can be easily separated from background images. It is worth noting that all the car images with a certain view do not necessarily coincide with one local region, as illustrated in Figure 1 where the car images of top view can belong to different local regions. Instead as long as these regions are localized in the feature space with small enough variances, each obtained local region will contain a relatively uniform view of car images. Better categorization performance can be obtained with smaller and easy-handled variances in these local regions by a divide-and-conquer manner. In other words, from the theoretical perspective of machine learning, the samples on each local region are approximately linear separable due to small within-class variations so that a simple model like linear classifier can have a satisfactory performance with a fast speed compared to other sophisticated algorithms (c.f. the results of simple linear LSSVM in Section 4.3).

A set of localized classifiers [2][4][15] exist in literatures, which leverage locality property to enhance the performance of classifiers. They gain great success and achieve state-of-the-art performance in many image classification tasks. However, their generalization performances are still restricted in twofold: (1) due to the small sample size on each local region, each local classifier is sensitive to noise which leads to overfitting phenomenon; (2) these local classifiers also ignore local correlation determined by the sample distribution in each local region. To overcome the above two problems, we first formulate and analyze a naive locality-sensitive classifier (LSC) by minimizing a locallyweighted loss function. By analyzing its generalization performance and drawback, we propose a novel locality- sensitive support vector machine (LSSVM) algorithm. This classifier applies locality-sensitive hashing (LSH) to divide the whole feature space into a number of local regions, on each of them a local model can be better constructed due to smaller within-class variation on it. To avoid these local models from overfitting into locality-sensitive structures, it imposes a global regularizer across local regions (c.f. Section 3) so that the local classifiers can be smoothly glued together to form a regularized overall classifier. Furthermore, local correlation is modeled to capture the sample distribution that determines the locality structure of each local region, which can increase the discriminating ability of LSSVM algorithm. By combining the global regularization and the correlation information in locality structure, the generalization performance of LSSVM can be significantly boosted as compared with the naive LSC.

The remainder of this paper is organized as follows. Section 2 proposes the framework for a naive locality-sensitive classifier, depicts its properties and analyzes its generalization performance. In Section 3, locality-sensitive support vector machine is specified which simultaneously exploits the local and global feature structures. Experiment results are shown in Section 4 on real-world web image data set. Finally we conclude in Section 5.

\section{A Naive Locality-Sensitive Classifier}

In this section, we propose a naive locality-sensitive classifier which forms the basis of the proposed algorithm in this paper. By analyzing its generalization performance and drawback, we show how these problems can be overcome by LSSVM in the following section.

\subsection{Background: Locality-Sensitive Hashing}

We start by a brief review of locality-sensitive hashing (LSH). The key idea is to assign a number to each sample in a metric space by a function $h$ uniformly selected from a family of hashing functions $\mathcal{H}$ so that the probability of collision (i.e., assigned by an identical number) is much higher for samples close to each other than those far apart [1]. For example, LSH in [7] uses a hashing function $h(\mathbf{x})=\left\llcorner\frac{\mathbf{a} \cdot \mathbf{x}+b}{r}\right\lrcorner$ to assign a hashing value to each sample $\mathbf{x}$, where $\mathbf{a}$ is a vector random variable with its entries randomly sampled from $p$-stable distribution, $b$ is a real number uniformly sampled from $[0, r]$ and $r$ controls the locality sensitivity (i.e., two samples within the distance $r$ have high probability of having the same hashing value). Then for any two samples $\mathbf{v}$ and $\mathbf{x}$ with $s$-norm distance in $d$-dimensional space $\mathbb{R}^{d}$, the probability of them having the same hashing 
value is

$$
\begin{aligned}
& \operatorname{Pr}(h(\mathbf{v})=h(\mathbf{x})) \\
& =\int_{0}^{r} \frac{1}{\|\mathbf{v}-\mathbf{x}\|_{s}} g_{p}\left(\frac{t}{\|\mathbf{v}-\mathbf{x}\|_{s}}\right)\left(1-\frac{t}{r}\right) d t \triangleq M(\mathbf{v}, \mathbf{x})
\end{aligned}
$$

where $g_{p}(\cdot)$ is the probability density function of the absolute value of the $s$-stable distribution, and $M(\mathbf{v}, \mathbf{x})$ is a nonnegative local weighting function having the peaking maximum value at $\mathbf{v}=\mathbf{x}$. In the following subsection, we show that the proposed locality-sensitive learner minimizes a loss function locally weighted by $M(\mathbf{v}, \mathbf{x})$ for the training samples $\mathbf{x}$ located in the local region around $\mathbf{v}$.

\subsection{Locality-Sensitive Classifier}

Given a set of training examples $S=$ $\left\{\left(\mathbf{x}_{j}, y_{j}\right) \mid j=1, \cdots, N\right\}$ and a candidate hypothesis space $\mathcal{F}$ from which the image classifier is learned, the conventional learning algorithm first constructs a loss function estimating the potential risk of misclassifying a testing sample and the optimal classifier is learned by minimizing this function. The most common loss function used in learning is the empirical loss function defined over $S$

$$
\mathcal{L}_{e m p}(f, S)=\frac{1}{N} \sum_{j=1}^{N} \ell\left(y_{j}, f\left(\mathbf{x}_{j}\right)\right)
$$

where $\ell(y, f(\mathbf{x}))$ is the loss function for a sample $(\mathbf{x}, y)$, e.g., $0 / 1 \operatorname{loss} \ell(y, f(\mathbf{x}))=\mathcal{I}(y \neq f(\mathbf{x}))$ or hinge loss $\ell(y, f(\mathbf{x}))=(1-y f(\mathbf{x}))_{+}$. Here $\mathcal{I}(E)$ is the indicator function taking value 1 if the event $E$ occurs or 0 otherwise and $(a)_{+}$is $a$ if it is positive or 0 otherwise.

Unlike the above conventional learning framework, the locality-sensitive learner attempts to minimize a loss function locally defined on a local region around an arbitrary testing sample $\boldsymbol{\nu}$. Such region is determined by hashing function uniformly sampled from $\mathcal{H}$. If the sampled hashing function $h$ has the same value on $\nu$ and $\mathbf{x}_{j}$, these two samples are close to each other in terms of property of hashing function. That is to say, $\mathbf{x}_{j}$ is in the locality-sensitive neighborhood of $\boldsymbol{\nu}$ and it is incorporated into the empirical locality-sensitive loss function for $\nu$. Formally, this loss function can be written as

$\mathcal{L}_{\text {emp }}(f, S, \boldsymbol{\nu})=\frac{1}{N} \sum_{j=1}^{N} \mathcal{I}\left(h(\boldsymbol{\nu})=h\left(\mathbf{x}_{j}\right)\right) \cdot \ell\left(y_{j}, f\left(\mathbf{x}_{j}\right)\right)$

where $h$ is sampled from $\mathcal{H}$. The optimal learner can be obtained

$$
f^{*}=\underset{f \in \mathcal{F}}{\arg \min } \mathcal{L}_{e m p}(f, S, \boldsymbol{\nu})
$$

The hashing function $h$ in formulation (3) equivalently divides the whole feature space $\mathcal{X}$ into a collection of disjoint $L$ regions $\mathcal{X}_{l}, l=1, \cdots, L$. As illustrated in Figure

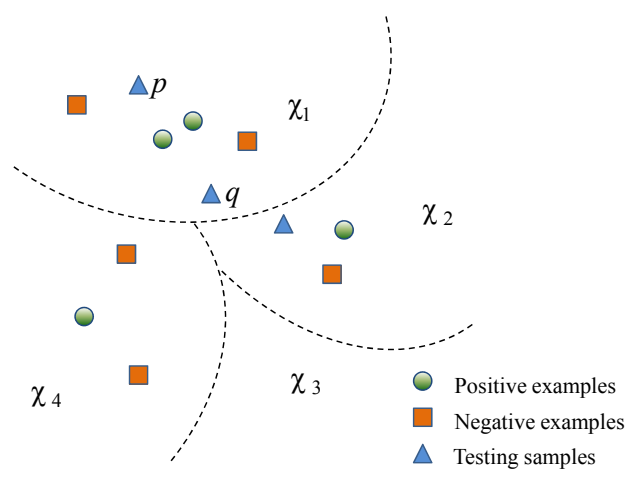

Figure 2. Testing sample $p$ and $q$ are both located in region $\mathcal{X}_{1}$ since $h(p)=h(q)$. Consequently, the obtained classifier by minimizing Eq. (3) for the testing samples in the same region is identical. That is to say, only one classifier need be solved for each region and all the testing sample in this region can be predicted by this classifier.

2 , testing sample $\mathbf{p}$ and $\mathbf{q}$ are both located in region $\mathcal{X}_{1}$ since $h(\mathbf{p})=h(\mathbf{q})$. Consequently, the obtained classifier by minimizing Eq. (3) for the testing samples in the same region is identical. That is to say, only one classifier need be solved for each region and all the testing sample in this region can be predicted by this classifier. The training of each local classifier only involves nearby training examples falling in the same local region with the same LSH value. We name this algorithm by locality-sensitive classifier (LSC) in this paper and summarize this naive LSC in Algorithm 1. Denote the classifier for region $\mathcal{X}_{l}$ by $f_{l}$, then for an arbitrary sample $\mathrm{x} \in \mathcal{X}$ the classifier is

$$
f(\mathbf{x})=\sum_{l=1}^{L} f_{l}(\mathbf{x}) \mathcal{I}\left(\mathbf{x} \in \mathcal{X}_{l}\right)
$$

The empirical loss in (3) is related to a kernel-based locally-weighted loss function [2] by computing its expectation with respect to the sampling distribution of hashing functions as Eq. (1). It is not difficult to verify the expected locally-weighted loss as

$$
\begin{aligned}
& \mathcal{L}_{0}(f, S, \boldsymbol{\nu})=\underset{h, S}{\mathbb{E}}\left[\mathcal{L}_{e m p}(f, S, \boldsymbol{\nu})\right] \\
& =\int_{\mathcal{X}} M\left(\boldsymbol{\nu}, \mathbf{x}_{j}\right) \cdot \ell(y, f(\mathbf{x})) d F_{\mathcal{D}}(\mathbf{x}, y)
\end{aligned}
$$

where $F_{\mathcal{D}}$ denotes the true sample distribution on $\mathcal{X}$. The proof of the above equality is given in Appendix A. We can find that $\mathcal{L}_{0}(f, S, \boldsymbol{\nu})$ weighs the samples nearer to $\boldsymbol{\nu}$ more heavily by the local weighting function $M(\boldsymbol{\nu}, \mathbf{x})$ and the learned classifier ought to have less loss in the local region around $\nu$, and a better classification accuracy for the testing sample $\nu$ can be expected. 


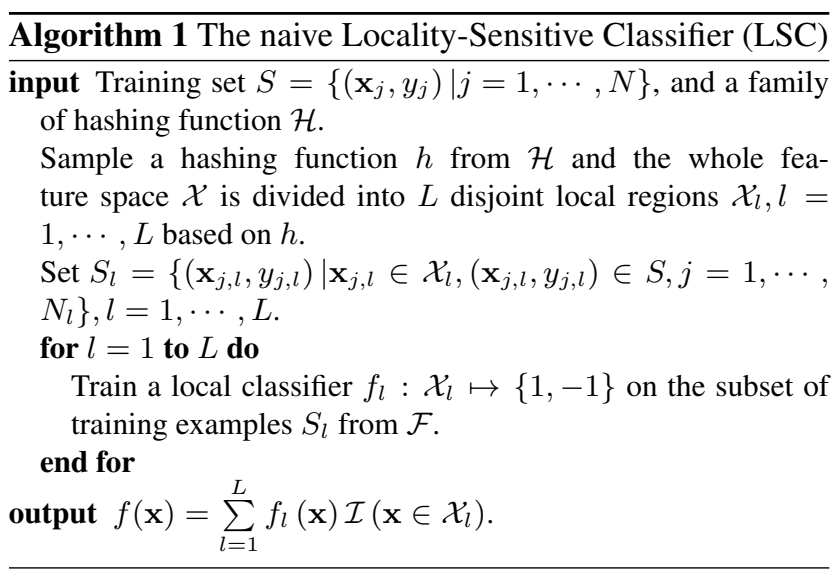

\subsection{Discussion}

Here, we give the generalization analysis of the overall LSC. According to (5) the local classifiers $f_{l}(\mathbf{x})$ are piecewise glued together to form the overall classifier $f$. This can result in irregular predictions near the boundary of two local regions so that the overall classifier is not smooth enough on the whole feature space. Such irregularity can degenerate the generalization performance and make the classifier sensitive to noises on each local region.

From the theoretical point of view, it is not difficult to see that the corresponding Vapnik-Chervonenkis (VC) dimension [13] of the LSC expressed by Eq. (5) is $L \cdot \mathcal{V C}(\mathcal{F})$ at most, where $L$ is the number of obtained local regions and $\mathcal{V C}(\mathcal{F})$ is the $\mathrm{VC}$ dimension of $\mathcal{F}$. High VC dimension can decrease the overall training error but increase the variance of the expected misclassifying rate. In other words, high VC dimension of LSC usually increases the risk of overfitting for the locality-sensitive classifier. To decrease the overfitting risk, in the next section we present a LocalitySensitive Support Vector Machine (LSSVM), in which the complexity of a strong learner with high VC dimension can be controlled by imposing extra smoothness requirement to avoid overfitting.

\section{Locality-Sensitive Support Vector Machine}

In this section we propose Locality-Sensitive Support Vector Machine (LSSVM) to overcome the drawback in native LSC. Suppose that the local classifier $f_{l}: \mathcal{X}_{l} \mapsto \mathcal{R}, l=$ $1, \cdots L$ on the $l$ th local region is a generalized hyperplane classifier

$$
f_{l}(\mathbf{x})=\left\langle\mathbf{w}_{l}, \mathbf{x}\right\rangle_{M_{l}}=\mathbf{w}_{l}^{T} M_{l} \mathbf{x}
$$

where $\mathbf{w}_{l}$ is the model parameter to be learned and $M_{l}$ is a semi-definite matrix which will be given later (c.f. Eq. (10)). Since individual local learner is limited on local region, the overall classifier (5) combining these local learners cannot guarantee the regularity on the whole space even though local learners have regularization performance on their own regions. Thus, we can assume there exists a global reference classifier $f(\mathbf{x})=\mathbf{w} \cdot \mathbf{x}$ on the whole space which is regularized to control its complexity as well as best approximates the local learners on each local region. Such a criterion can be implemented by introducing the following regularization term on each local region $\mathcal{X}_{l}$ as

$$
\begin{aligned}
& \Omega\left(\mathbf{w}, \mathbf{w}_{l}\right)=\int_{\mathcal{X}_{l}}\left|\mathbf{w}_{l} \cdot \mathbf{x}-\mathbf{w} \cdot \mathbf{x}\right|^{2} d F_{l}(\mathbf{x}) \\
& \doteq \frac{1}{N_{l}} \sum_{j=1}^{N_{l}}\left(\mathbf{w} \cdot \mathbf{x}_{j, l}-\mathbf{w}_{l} \cdot \mathbf{x}_{j, l}\right)^{2} \\
& =\frac{1}{N_{l}} \sum_{j=1}^{N_{l}}\left(\mathbf{w}-\mathbf{w}_{l}\right)^{T} \mathbf{x}_{j, l} \cdot \mathbf{x}_{j, l}^{T}\left(\mathbf{w}-\mathbf{w}_{l}\right) \\
& =\left(\mathbf{w}-\mathbf{w}_{l}\right)^{T} X_{l} X_{l}^{T}\left(\mathbf{w}-\mathbf{w}_{l}\right) \\
& =\left(\mathbf{w}-\mathbf{w}_{l}\right)^{T} S_{l}\left(\mathbf{w}-\mathbf{w}_{l}\right)
\end{aligned}
$$

where $N_{l}$ is the number of training examples on the $l$ th region, $\mathbf{x}_{j, l}$ is the $j$ th example in the $l$ th region, $X_{l}$ is the matrix with $\mathbf{x}_{j, l}, j=1, \cdots, N_{l}$ as its columns, and $S_{l}=\frac{1}{N_{l}} \sum_{j=1}^{N_{l}} \mathbf{x}_{j, l} \cdot \mathbf{x}_{j, l}^{T}$ is sample correlation matrix for the $l$ th region. Note that in the second step above, we use the sample frequencies as the weighting factors to approximate the true distribution $F_{l}(\mathrm{x})$ on each region. The local correlation in (8) plays the key role in connecting the local classifiers to the global reference classifier, and it serves to regularize the local classifier with the global structure on the whole feature space. With the above regularizer on each region, the learning problem for locality-sensitive classifier can be formulated as

$$
\begin{aligned}
& \min _{\mathbf{w}, \mathbf{w}_{l}} \frac{1}{2} \lambda\|\mathbf{w}\|_{2}^{2}+\frac{1}{2} \sum_{l=1}^{L}\left(\mathbf{w}-\mathbf{w}_{l}\right)^{T} S_{l}\left(\mathbf{w}-\mathbf{w}_{l}\right) \\
& +C \sum_{l=1}^{L} \sum_{j=1}^{N_{l}} \xi_{j, l} \\
& \text { s.t., } y_{j, l} \cdot \mathbf{w}_{l}^{T} S_{l} \mathbf{x}_{j, l} \geq 1-\xi_{j, l}, \xi_{j, l} \geq 0, j=1, \cdots, N_{l}, \\
& l=1, \cdots, L
\end{aligned}
$$

where $y_{j, l} \in\{1,-1\}$ is the corresponding class label for each $\mathbf{x}_{j, l}$, and $\lambda$ and $C$ are the balance parameters. Note that here we substitute $S_{l}$ for $M_{l}$ in Eq. (7) to form the local classifier $f_{l}$. Since the correlation matrix $S_{l}$ is positive semi-definite matrix so that it can be can be factorized into $S_{l}=Q_{l}^{T} Q_{l}$ where $Q_{l} \in \mathbb{R}^{d^{\prime} \times d}$ with $d^{\prime}=\operatorname{Rank}\left(S_{l}\right)$. The corresponding local classifier $f_{l}$ can be written as

$$
f_{l}(\mathbf{x})=\mathbf{w}_{l}^{T} S_{l} \mathbf{x}=\mathbf{w}_{l}^{T} Q_{l}^{T} Q_{l} \mathbf{x}=\left(Q_{l} \mathbf{w}_{l}\right)^{T}\left(Q_{l} \mathbf{x}\right)
$$

so $f_{l}$ can be seen as a linear classifier learned on a localized subspace transformed from $\mathbb{R}^{d}$ by $Q_{l}$. The dimensionality of this subspace is $\operatorname{Rank}\left(Q_{l}\right)=\operatorname{Rank}\left(S_{l}\right)$ which does not necessarily agree with that of its ambient space $\mathbb{R}^{d}$ but can be much smaller than $d$. For example, imagine a local region with $N_{l}$ samples in a relatively high dimensional 
ambient space $\mathbb{R}^{d}$ where $N_{l} \ll d$. The local dimensionality is at most $\operatorname{Rank}\left(X_{l}\right)$ because the intrinsic localized subspace is spanned by these $N_{l}$ samples whose rank equals to $\operatorname{Rank}\left(S_{l}\right)$ since $S_{l}=X_{l} X_{l}^{T}$ (In Eq. (10), we will explicitly make an embedding of the local samples into this localized subspace). In other words, even in a high dimensional space, the local region can still have an intrinsically low dimensionality. Such phenomenon is conspicuous in the local region due to the rare sample problem, i.e., all the training examples are assigned to different regions and each region may only contain a small portion. Therefore by working in the underlying subspace spanned by these samples, the classifier can be more compact with fewer parameters. This compact classifier also alleviates the rare sample problem based on PAC (Probably Approximately Correct)-learnable model [11]. Because when learning with it, the number of training samples which suffice to learn a local classifier $f_{l}$ with an error rate at most $\epsilon$ can be reduced to $\mathcal{O}\left(\operatorname{Rank}\left(S_{l}\right)\right)$, as compared with $\mathcal{O}(d)$ when learning in the ambient space $\mathbb{R}^{d}$ where $\operatorname{Rank}\left(S_{l}\right) \leq N_{l} \ll d$.

Formulating the Lagrangian function for Problem (8) and maximizing it over the original variables [3], the corresponding dual problem can be written as

$$
\begin{aligned}
& \max _{\alpha_{j, l}} \sum_{l=1}^{L} \sum_{j=1}^{N_{l}} \alpha_{j, l}-\frac{1}{2} \sum_{l=1}^{L} \sum_{m=1}^{L} \sum_{j=1}^{N_{l}} \sum_{i=1}^{N_{m}} \alpha_{j, l} \alpha_{i, m} \\
& \cdot\left\{\frac{1}{\lambda} \mathbf{x}_{j, l}^{T} S_{l}^{T} S_{m} \mathbf{x}_{i, m}+\delta_{l, m} \mathbf{x}_{j, l}^{T} S_{l} \mathbf{x}_{i, m}\right\} \\
& \text { s.t., } 0 \leq \alpha_{j, l} \leq C
\end{aligned}
$$

The derivation of this dual problem is given in Appendix B. This linear formulation can be extended into kernelized nonlinear version by introducing a mapping $g$ from the input space $\mathbb{R}^{d}$ to another space $\mathcal{U}$ with the inner product $g(\mathbf{x}) \cdot g(\mathbf{y})=k(\mathbf{x}, \mathbf{y})$ as kernel function. Define $X_{l}=\frac{1}{\sqrt{N_{l}}}\left[g\left(\mathbf{x}_{1, l}\right), \cdots g\left(\mathbf{x}_{N_{l}, l}\right)\right]$ is the matrix with each mapped sample in the $l$ th region as its column vector, we have $S_{l}=X_{l} X_{l}^{T}$. Therefore it is not difficult to find that the dual problem (11) can be rewritten as

$$
\begin{aligned}
& \max _{\alpha_{j, l}} \sum_{l=1}^{L} \sum_{j=1}^{N_{l}} \alpha_{j, l}-\frac{1}{2} \sum_{l=1}^{L} \sum_{m=1}^{L} \sum_{j=1}^{N_{l}} \sum_{i=1}^{N_{m}} \alpha_{j, l} \alpha_{i, m} \\
& \cdot\left\{\frac{1}{\lambda N_{l} N_{m}} \mathbf{k}_{j, l}^{T} K_{l, m} \mathbf{k}_{i, m}+\frac{\delta_{l, m}}{N_{l}} \delta_{l, m} \mathbf{k}_{j, l}^{T} \mathbf{k}_{i, m}\right\} \\
& \text { s.t., } 0 \leq \alpha_{j, l} \leq C
\end{aligned}
$$

where

$$
\begin{aligned}
& \mathbf{k}_{j, l}=\left[k\left(\mathbf{x}_{j, l}, \mathbf{x}_{1, l}\right), \cdots k\left(\mathbf{x}_{j, l}, \mathbf{x}_{N_{l}, l}\right)\right]^{T} \\
& \mathbf{k}_{i, m}=\left[k\left(\mathbf{x}_{i, m}, \mathbf{x}_{1, m}\right), \cdots k\left(\mathbf{x}_{i, m}, \mathbf{x}_{N_{m}, m}\right)\right]^{T}
\end{aligned}
$$

are column vectors containing the kernel functions of $\mathbf{x}_{j, l}$, $\mathbf{x}_{i, m}$ and the samples in their respective local regions. And

$$
K_{l, m}=\left[k\left(\mathbf{x}_{j, l}, \mathbf{x}_{i, m}\right)\right]_{N_{l} \times N_{m}}
$$

is the kernel matrix between all the samples in the $l$ th and the $m$ th regions.

Finally, when the coefficients $\alpha_{j, l}$ are solved the local classifiers are given as

$$
\begin{aligned}
& f_{t}(\mathbf{x})=\sum_{l=1}^{L} \sum_{j=1}^{N_{l}} \alpha_{j, l}\left\{\frac{1}{\lambda N_{l} N_{t}} \mathbf{k}_{j, l}^{T} K_{l, t} \mathbf{k}_{t}(\mathbf{x})+\frac{\delta_{l, t}}{N_{l}} \mathbf{k}_{j, l}^{T} \mathbf{k}_{t}(\mathbf{x})\right\} \\
& 1 \leq t \leq L
\end{aligned}
$$

where $\mathbf{k}_{t}(\mathbf{x})=\left[k\left(\mathbf{x}_{1, t}, \mathbf{x}\right), \cdots k\left(\mathbf{x}_{N_{t}, t}, \mathbf{x}\right)\right]^{T}$. Note that the sample correlation matrices $S_{l}$ 's are used to construct the local classifiers $f_{l}$ 's based on Eq. (10). However, if a testing sample belongs to a region without any training example, its corresponding $S_{l}$ does not exist. In this case, we can use the global reference classifier $f(\mathbf{x})=\mathbf{w}^{T} \mathbf{x}$ to predict it. By letting $S_{l}=I$ in Eq. (19), the global reference classifier can be computed as $f(\mathbf{x})=\mathbf{w}^{T} \mathbf{x}$. Therefore by substituting $S_{l}$ with the identity matrix $I$ in this case, the algorithm still works in the same way. At this time, the predicting function of Eq. (15) changes to be

$$
\begin{aligned}
& f(\mathbf{x})=\sum_{l=1}^{L} \sum_{j=1}^{N_{l}} \frac{\alpha_{j, l}}{\lambda} g\left(\mathbf{x}_{j, l}\right)^{T} S_{l}^{T} g(\mathbf{x}) \\
& =\sum_{l=1}^{L} \sum_{j=1}^{N_{l}} \frac{\alpha_{j, l}}{\lambda} g\left(\mathbf{x}_{j, l}\right)^{T} X_{l} X_{l}^{T} g(\mathbf{x}) \\
& =\sum_{l=1}^{L} \sum_{j=1}^{N_{l}} \frac{\alpha_{j, l}}{\lambda N_{l}} \mathbf{k}_{j, l}^{T} K_{l, t} \mathbf{k}_{l}(x)
\end{aligned}
$$

\subsection{Discussion}

- Global regularization. Considering an extreme case when $\lambda=0$, it is not difficult to find the formulation in Eq. (18) is factorized into $L$ independent problems defined on $L$ local regions $\mathcal{X}_{l}$ for $l=1, \cdots, L$. In this extreme case the global regularization term disappears and the learned local classifiers have overfitting risk due to relatively small number of examples in local region. On the contrary, introducing the global regularization term serves to relieve the overfitting effect and improve the robustness of these local classifiers. So the learned classifier preserves both its locality characteristic and improves its generalization ability on the global feature space.

- Connection with Multi-Task Learning. If each local classifier is seen as an individual task, LSSVM is connected with multi-task learning [8], and the global reference function can be seen as the shared classification structure among individual classifiers. However, the proposed LSSVM differs from the conventional multi-task learning algorithm in that the local correlation $S_{l}$ is incorporated in $\Omega\left(\mathbf{w}, \mathbf{w}_{l}\right)$ to capture the sample distribution in each local region. As stated in the above subsection, it naturally measures the distinction between the local classifier and the global refer- 
ence function along the axes transformed by $Q_{l}$ from the correlation matrix $S_{l}=Q_{l}^{T} Q_{l}$. In other words, it takes into account the feature correlation of all the examples in each local region and is scale-invariant so that the global reference function can approximate each local classifier adaptively. Thus, the local correlation has powerful discriminating ability to capture the structure of each individual local region.

\section{Experiments}

In this section, we conduct experiments to compare the proposed LSSVM with other state-of-the-art localized classifiers such as KNN-SVM [15] and localized support vector machine (LocSVM)[4] as well as the conventional support vector machine (SVM) [13]. To validate the effect of local correlation in the regularizer (8), we also construct LSSVM with no local correlation (LSSVM-NLC) by replacing each local correlation matrix $S_{l}$ with the identity matrix.

- First, we conduct experiments on a benchmark data set with two-dimensional feature vectors (c.f. Section 4.1). It can give an intuitive example on how LSSVM works on local structures and how the locality-sensitivity parameters affect their performances.

- Next, the algorithms are tested on a real-world image data set NUS-WIDE-SCENE collected from Flickr with 33 different image categories (c.f. Section 4.2).

- Moreover, we show a competitive result obtained by a linear LSSVM model whose local learner is a simple linear classifier. It shows such a simple classifier can be boosted significantly under the localitysensitive framework discussed in Section 3.
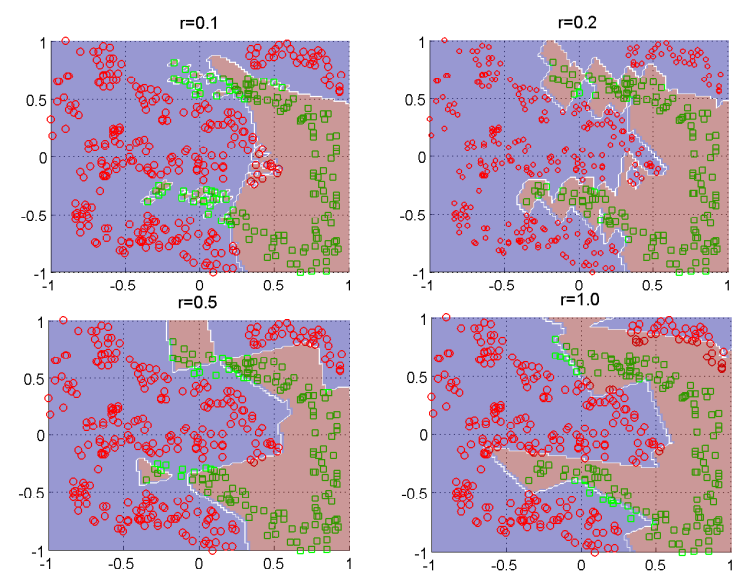

Figure 3. Learned classification boundaries of LSSVM with different locality-sensitive parameters $r$ 's.

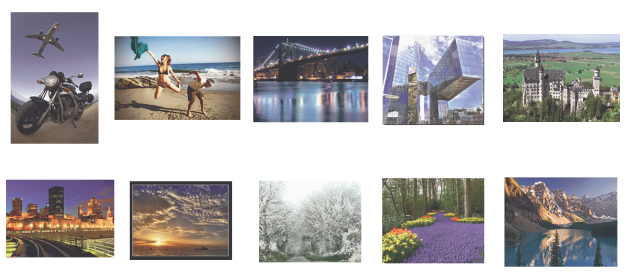

Figure 4. Illustration of example images collected from Flickr.com.

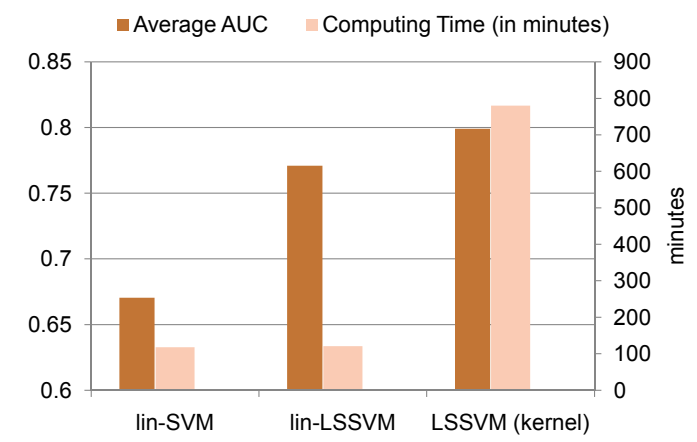

Figure 5. Comparison of lin-LSSVM with the conventional linear support vector machine (lin-SVM), LSSVM in terms of average AUC.

\subsection{Experiment on Benchmark data set}

To illustrate intuitive classification results of the proposed algorithms, we conduct experiments on a publiclyavailable two-dimensional data set ever used in [10]. First we use half of the data set to train the LSSVM, and obtain the corresponding classification results in Figure 3. Different locality-sensitive parameters $r$ 's are used to learn these four different results. As shown, when $r$ is smaller, such as $r=0.1$ and $r=0.2$ the learned classifiers learn much fine classification boundaries on the local structures. While $r$ increases, the global structures are learned better but smaller local structures (i.e., these isolated points) are identified as noisy outliers and neglected. Such results are consistent with the theoretical analysis in Section 3, where a globallocal learning strategy is introduced to balance the knowledge in the local and global structures. Experiments show that by choosing a proper $r$, the classification accuracy can achieve $99.77 \%$ in this case.

\subsection{Experiment on Flickr data set}

We also compare these algorithms on a public realworld image data set NUS-WIDE-SCENE [5] collected from Flickr (See Figure 4 for some example images). This data set contains 17,463 images and 33 different associated concepts. Three types of features are extracted from each images: 73 dimensional edge direction histogram, 128 dimensional wavelet texture and 225 dimensional block-wise 
Table 1. Comparison of SVM, SVM-KNN, LocSVM, LSSVM-NLC and LSSVM on the Flickr data set in terms of AUC.

\begin{tabular}{|c|c|c|c|c|c|c|c|c|c|c|c|}
\hline Concept & SVM & SVM-KNN & LocSVM & LSSVM-NLC & LSSVM & Concept & SVM & SVM-KNN & LocSVM & LSSVM-NLC & LSSVM \\
\hline airport & 0.7389 & 0.7442 & 0.7721 & 0.8137 & 0.8384 & ocean & 0.6726 & 0.6796 & 0.6734 & 0.7193 & 0.7588 \\
\hline beach & 0.6561 & 0.6704 & 0.6490 & 0.7128 & 0.7493 & plants & 0.7241 & 0.7299 & 0.7372 & 0.7569 & 0.7793 \\
\hline bridge & 0.5735 & 0.5773 & 0.5871 & 0.6189 & 0.6479 & railroad & 0.7245 & 0.7192 & 0.7737 & 0.7661 & 0.8096 \\
\hline buildings & 0.6552 & 0.6520 & 0.6666 & 0.6919 & 0.7287 & rainbow & 0.7495 & 0.7433 & 0.7633 & 0.781 & 0.8304 \\
\hline castle & 0.7043 & 0.7022 & 0.7449 & 0.7629 & 0.8035 & reflection & 0.6718 & 0.6819 & 0.7032 & 0.7266 & 0.7644 \\
\hline cityscape & 0.7160 & 0.7062 & 0.7151 & 0.7411 & 0.8282 & road & 0.6134 & 0.6086 & 0.6459 & 0.6503 & 0.6826 \\
\hline clouds & 0.7165 & 0.7294 & 0.7386 & 0.7005 & 0.8039 & sky & 0.7114 & 0.7242 & 0.7435 & 0.7709 & 0.8047 \\
\hline frost & 0.7359 & 0.7465 & 0.7370 & 0.7514 & 0.8069 & snow & 0.7442 & 0.7579 & 0.7502 & 0.7894 & 0.8079 \\
\hline garden & 0.8266 & 0.8356 & 0.8403 & 0.8056 & 0.9040 & street & 0.7757 & 0.7855 & 0.7730 & 0.8204 & 0.8677 \\
\hline glacier & 0.6991 & 0.7215 & 0.7119 & 0.7122 & 0.8313 & sunset & 0.7428 & 0.7646 & 0.7424 & 0.8121 & 0.8565 \\
\hline grass & 0.8119 & 0.8264 & 0.8442 & 0.8063 & 0.8918 & temple & 0.6675 & 0.6590 & 0.7136 & 0.6987 & 0.7341 \\
\hline harbor & 0.6863 & 0.6804 & 0.7361 & 0.6721 & 0.8136 & town & 0.7388 & 0.7329 & 0.7568 & 0.7893 & 0.8327 \\
\hline house & 0.6882 & 0.6924 & 0.7390 & 0.6842 & 0.7792 & valley & 0.7591 & 0.7728 & 0.7736 & 0.8344 & 0.8625 \\
\hline lake & 0.6344 & 0.6406 & 0.6531 & 0.6267 & 0.7271 & water & 0.6191 & 0.6372 & 0.6466 & 0.66 & 0.6882 \\
\hline moon & 0.7050 & 0.7184 & 0.7253 & 0.6739 & 0.7879 & waterfall & 0.8495 & 0.8612 & 0.8567 & 0.9011 & 0.9404 \\
\hline mountain & 0.6865 & 0.7043 & 0.6976 & 0.7513 & 0.7811 & window & 0.6799 & 0.6777 & 0.6871 & 0.7021 & 0.7257 \\
\hline nighttime & 0.8199 & 0.8214 & 0.8414 & 0.8538 & 0.9000 & & & & & & \\
\hline
\end{tabular}

color moments. The data set is divided into the training and testing sets. The models are trained with Gaussian kernels. All training parameters are divided into two types. (1) The parameters regarding local classifier defined in Section 3, including $\lambda, C$ and the radius width $\sigma$ in Gaussian kernels. They are tuned and selected by a two-fold cross validation procedure on the training set. (2) The parameters to form the local regions. They are fixed without tuning across different concepts. Respectively, 512 locality-sensitive regions are finally obtained in LSSVM. Note that the LSH procedure is repeated three times to enlarge the gap between the probabilities of collision for close and far samples [1]. Also, for simplicity a modulo operation is applied to each hashing value to obtain a fix-sized hash table which still preserve the locality sensitivity.

All algorithms are compared by measuring their retrieval performances based on AUC metrics (i.e., area under the ROC curve) [9]. The ROC curve plots the true positive rate as a function of the false positive rate, and AUC measures the probability that a randomly chosen positive image will be ranked higher than a randomly chosen negative image.

Results We compare the performances between SVM, SVM-KNN, LocSVM, LSSVM-NLC and the proposed LSSVM in Table 1. It is shown that LSSVM outperforms the other algorithms on all the 33 concepts. Especially, LSSVM outperforms LSSVM-NLC which is a version of LSSVM with no local correlation information. This confirms our assertion that local correlation can help LSSVM to improve the generalization performance by capturing the locality structure determined by the sample distribution in each local region.

\subsection{Results of A Simple Linear Model}

Furthermore, as stated in Section 2.3, the proposed locality-sensitive algorithm can increase the capacity (i.e., the VC dimension) of the original local learner, so we expect the performance of a simple local classifier can be boosted in this framework. Here we test this assumption by using a linear classifier as the local learner instead of kernelized support vector machine with Gaussian kernel in the above. We denote this linear version of LSSVM by linLSSVM. The results of average AUC on 33 concepts are shown in Figure 5. As compared with the conventional linear SVM (lin-SVM), we find lin-LSSVM has obtained a significantly better performance which is comparable with the above LSSVM (kernel) with Gaussian kernels. This result is greatly useful to many practical applications since the simple linear model has much fewer parameters to be learned compared to their kernelized counterparts so that it can be trained and predicted rapidly. Also the linear model will not swell like the kernelized SVMs whose support vector numbers will increase rapidly when training examples increases. As illustrated in Figure 5, the computing time used by lin-LSSVM for training and prediction over all 33 concepts is only about one eighth of their kernelized counterparts.

\section{Conclusion}

In this paper, we propose a locality-sensitive classifier to model the local region with smaller intra-class variation by exploring the local correlation structure. A global regularization is proposed to avoid overfitting risk due to rare sample problem in the local regions. It smoothly glues the local classifiers by adapting from a global reference function. Furthermore, it takes into account the feature correlations on each local region and is scale-invariant so that the global reference function can adaptively approximate each local classifier. This can increase the discriminating ability of the local classifiers on individual locality regions. Experiments on real-world web image data set show the competitive results obtained by LSSVM. The results also show that even linear version of LSSVM can enhance the performance of linear classifier without incorporating computing-intensive kernel function. 


\section{Appendix A}

Proof of Equation (6):

$$
\begin{aligned}
& \underset{h, S}{\mathbb{E}}\left[\mathcal{L}_{e m p}(f, S, \boldsymbol{\nu})\right] \\
= & \underset{S}{\mathbb{E}}\left[\underset{h}{\mathbb{E}}\left[\mathcal{L}_{e m p}(f, S, \boldsymbol{\nu}) \mid S\right]\right] \\
= & \underset{S}{\mathbb{E}}\left[\frac{1}{N} \sum_{j=1}^{N} \underset{h}{\mathbb{E}}\left[\mathcal{I}\left(h(\boldsymbol{\nu})=h\left(\mathbf{x}_{j}\right)\right) \cdot \ell\left(y_{j}, f\left(\mathbf{x}_{j}\right)\right) \mid S\right]\right] \\
= & \underset{S}{\mathbb{E}}\left[\frac{1}{N} \sum_{j=1}^{N} \underset{h}{\mathbb{E}}\left[\mathcal{I}\left(h(\boldsymbol{\nu})=h\left(\mathbf{x}_{j}\right)\right) \mid S\right] \cdot \ell\left(y_{j}, f\left(\mathbf{x}_{j}\right)\right)\right] \\
= & \underset{S}{\mathbb{E}}\left[\frac{1}{N} \sum_{j=1}^{N} M\left(\boldsymbol{\nu}, \mathbf{x}_{j}\right) \cdot \ell\left(y_{j}, f\left(\mathbf{x}_{j}\right)\right)\right] \\
= & \int_{\mathcal{X}} M(\boldsymbol{\nu}, \mathbf{x}) \cdot \ell(y, f(\mathbf{x})) d F_{\mathcal{D}}(\mathbf{x}, y)
\end{aligned}
$$

The first equality of the above equation follows the iterated law of conditional expectation.

\section{Appendix B}

Derivation of dual problem (11).

Let $\mathbf{v}_{l}=Q_{l}\left(\mathbf{w}_{l}-\mathbf{w}\right)$, we have

$$
\begin{aligned}
& \mathbf{w}_{l}^{T} S_{l} \mathbf{x}=\mathbf{w}_{l}^{T} Q_{l}^{T} Q_{l} \mathbf{x}=\left(\mathbf{v}_{l}+Q_{l} \mathbf{w}\right)^{T} Q_{l} \mathbf{x} \\
& =\mathbf{v}_{l}^{T} Q_{l} \mathbf{x}+\mathbf{w}^{T} Q_{l}^{T} Q_{l} \mathbf{x}=\mathbf{v}_{l}^{T} Q_{l} \mathbf{x}+\mathbf{w}^{T} S_{l} \mathbf{x}
\end{aligned}
$$

Therefore Eq.(9) can be written as

$$
\begin{aligned}
& \min _{\mathbf{w}, \mathbf{w}_{l}} \frac{1}{2} \lambda\|\mathbf{w}\|_{2}^{2}+\frac{1}{2} \sum_{l=1}^{L}\left\|\mathbf{v}_{l}\right\|_{2}^{2}+C \sum_{l=1}^{L} \sum_{j=1}^{N_{l}} \xi_{j, l} \\
& \text { s.t., } y_{j, l}\left(\mathbf{v}_{l}^{T} Q_{l} \mathbf{x}_{j, l}+\mathbf{w}^{T} S_{l} \mathbf{x}_{j, l}\right) \geq 1-\xi_{j, l}, \\
& \xi_{j, l} \geq 0, j=1, \cdots, N_{l}, l=1, \cdots, L
\end{aligned}
$$

Define the feature mapping

$$
\begin{aligned}
& \varphi_{l}(\mathbf{x}) \\
& =[\frac{1}{\sqrt{\lambda}}\left(S_{l} \mathbf{x}\right)^{T}, \underbrace{0, \cdots, 0}_{l-1}, \quad\left(Q_{l} \mathbf{x}\right)^{T}, \underbrace{0, \cdots, 0}_{L-l}]^{T} \\
& \in \mathbb{R}^{d+\sum_{l=1}^{L} \operatorname{Rank}\left(S_{l}\right)}
\end{aligned}
$$

and weighting vector

$$
\Xi=\left[\begin{array}{llll}
\sqrt{\lambda} \mathbf{w}^{T} & \mathbf{v}_{1}^{T} & \cdots & \mathbf{v}_{L}^{T}
\end{array}\right]^{T} \in \mathbb{R}^{d+\sum_{l=1}^{L} \operatorname{Rank}\left(S_{l}\right)}
$$

The local classifier for the $l$ th region can be written as

$$
f_{l}(\mathbf{x})=\Xi \cdot \varphi_{l}(\mathbf{x})=\mathbf{w}_{l}^{T} S_{l} \mathbf{x}
$$

and

$$
\begin{aligned}
& \|\Xi\|^{2}=\lambda\|\mathbf{w}\|^{2}+\sum_{l=1}^{L}\left\|\mathbf{v}_{l}\right\|^{2} \\
& =\lambda\|\mathbf{w}\|^{2}+\sum_{l=1}^{L}\left(\mathbf{w}_{l}-\mathbf{w}\right)^{T} S_{l}\left(\mathbf{w}_{l}-\mathbf{w}\right)
\end{aligned}
$$

With the above notations, problem (18) can be rewritten as

$$
\begin{aligned}
& \min _{\mathbf{w}, \mathbf{w}_{l}} \frac{1}{2}\|\Xi\|^{2}+C \sum_{l=1}^{L} \sum_{j=1}^{N_{l}} \xi_{j, l} \\
& \text { s.t., } y_{j, l} \Xi \cdot \varphi_{l}\left(\mathbf{x}_{j, l}\right) \geq 1-\xi_{j, l}, \\
& \xi_{j, l} \geq 0, j=1, \cdots, N_{l}, l=1, \cdots, L
\end{aligned}
$$

Since the inner product between the mapped features $\varphi_{l}\left(\mathbf{x}_{j, l}\right)$ and $\varphi_{m}\left(\mathbf{x}_{i, m}\right)$ as in Eq. (19) is $\varphi_{l}\left(\mathbf{x}_{j, l}\right)$. $\varphi_{m}\left(\mathbf{x}_{i, m}\right)=\frac{1}{\lambda} \mathbf{x}_{j, l}^{T} S_{l}^{T} S_{m} \mathbf{x}_{i, m}+\delta_{l, m} \mathbf{x}_{j, l}^{T} S_{l} \mathbf{x}_{i, m}$, it follows that the dual problem (11) can be derived directly from (23).

\section{References}

[1] A. Andoni and P. Indyk. Near-optimal hashing algorithms for approximate nearest neighbor in high dimensions. Communications of the ACM, (1), January 2008. 842, 847

[2] L. Bottou and V. Vapnik. Local learning algorithms. Neural Computation, (6):888-900, November 1992. 842, 843

[3] S. Boyd and L. Vandenberghe. Convex Optimization. Cambridge University Press, 2004. 845

[4] H. Cheng, P.-N. Tan, and R. Jin. Localized support vector machine and its efficient algorithm. In Proc of SIAM Int'l Conf. on Data Mining, 2007. 842, 846

[5] T.-S. Chua, J. Tang, R. Hong, H. Li, Z. Luo, and Y.-T. Zheng. Nus-wide: A real-world web image database from national university of singapore. In Proc. ACM CIVR, 2009. 846

[6] O. Chum, M. Perd'och, and J. Matas. Geometric minhashing: Finding a (thick) needle in a haystack. In Proc. IEEE CVPR, 2009. 841

[7] M. Datar, N. Immorlica, P. Indyk, and V. S. Mirrokni. Locality-sensitive hashing scheme based on p-stable distributions. In Proc. ACM SCG, Brooklyn, New York, June 9-11 2004. 842

[8] T. Evgeniou and M. Pontil. Regularized multi-task learning. In Proc. SIGKDD, 2004. 845

[9] J. A. Hanley and B. J. McNeil. The meaning and use of the area under a receiver operating characteristic (roc) curve. Radiology, 143:29-36, 1982. 847

[10] T. K. Ho and E. M. Kleinberg. Building projectable classifiers of arbitrary complexity. In Proc. ICPR, 1996. 846

[11] T. Mitchell. Machine Learning, chapter Computational Learning Theory. McGraw Hill, 1997. 845

[12] G.-J. Qi, X.-S. Hua, and H.-J. Zhang. Learning semantic distance from community-tagged media collection. In Proc. ACM Multimedia, 2009. 841

[13] V. N. Vapnik. Statistical Learning Theory. John Wiley \& Sons, Inc, 1998. 844, 846

[14] Z. Wu, Q. Ke, M. Isard, and J. Sun. Bundling features for large scale partial-duplicate web image search. In Proc. IEEE CVPR, 2009. 841

[15] H. Zhang, A. C. Berg, M. Maire, and J. Malik. Svm-knn: Discriminative nearest neighbor classification for visual category recognition. In Proc. IEEE CVPR, 2006. 842, 846 